\title{
CLINICAL CASE REPORT
}

A case report suggestive of strongyloidiasis infection occurring in temperate Australia

AUTHOR

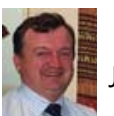

John Fraser ${ }^{1}$ MD, Adjunct Professor *

\section{CORRESPONDENCE}

* John Fraser jfrase22@une.edu.au

\section{AFFILIATIONS}

${ }^{1}$ School of Rural Medicine, University of New England, Armidale, NSW 2531, Australia

\section{PUBLISHED}

15 May 2019 Volume 19 Issue 2

\section{HISTORY}

RECEIVED: 13 February 2018

REVISED: 2 April 2019

ACCEPTED: 8 April 2019

\section{CITATION}

Fraser J. A case report suggestive of strongyloidiasis infection occurring in temperate Australia. Rural and Remote Health 2019; $19: 4787$. https://doi.org/10.22605/RRH4787

ETHICS APPROVAL: Consent to publish this case report in a refereed journal was obtained from the patient.

Except where otherwise noted, this work is licensed under a Creative Commons Attribution 4.0 International Licence 


\section{ABSTRACT:}

Strongyloides stercoralis is a nematode that can cause fatal systemic or disseminated infections in immunocompromised persons. It is known to be endemic in tropical Australia. Sporadic cases arising from temperate regions are reported in Russia and North America. An Aboriginal woman aged 71 years with ovarian carcinoma developed worsening lethargy and tiredness. She was diagnosed with strongyloidiasis based on serology in 2015. She had resided in the state of New South Wales all her life. This case report provides further evidence that strongyloides transmission is possible in temperate areas of Australia and has occurred in the past when sanitation was not as advanced as it is today.

\section{Keywords:}

Aboriginal health, Australia, immunosuppression, infectious diseases, parasitology, Strongyloides, temperate, tropical.

\section{FULL ARTICLE:}

\section{Introduction}

Strongyloides stercoralis is a nematode living predominantly in the intestines of humans, with some stages developing outside the body. These environmental stages are reliant on faecal contamination of warm, moist soil ${ }^{\mathbf{1}}$. Consequently, it can be considered to be a disease of socioeconomic disadvantage associated with poor sanitation ${ }^{\mathbf{1}}$. The life cycle is depicted in Figure 1.

Within the soil, male and female 'free living' worms mate to form new fertile eggs. These eggs develop initially into early rhabditiform larvae, developing into filariform larvae, which penetrate skin. Only one free-living cycle can occur in the soil before a new human host is found ${ }^{\mathbf{2}, 3}$.

After skin penetration, the larvae migrate into capillaries to enter the venous circulation to return to the lung. There they are coughed and swallowed to enter the bowel, where the worm can mature. The process of parthogenesis means an individual adult parasitic female worm can replicate new generations of viable eggs within the human host without contact with other worms or the soil ${ }^{\mathbf{1}}$. These viable eggs can mature in the intestine with subsequent larval penetration of the lower rectum. Autoinfection occurs with larval re-entry to the venous circulation, taking larvae to the lung. Random migration to other organs may also occur. The patient's immune system can keep Strongyloides numbers at a relative stable number. The vast majority of patients with positive S. stercoralis serology are asymptomatic.

A change, however, in a person's immunity can cause asymptomatic strongyloidiasis to progress to fulminant systemic or disseminated disease, where deaths can occur, often decades after initial infection $\mathbf{4 , 5}$

This case is presented to highlight the possibility that strongyloidiasis infection can occur in temperate regions of Australia. This transmission is likely to have occurred years ago when sanitation was not as well developed as it is today.

Consent to publish this case report in a refereed journal was obtained from the patient. She hoped others would benefit from highlighting awareness of strongyloidiasis in the immunocompromised patient.

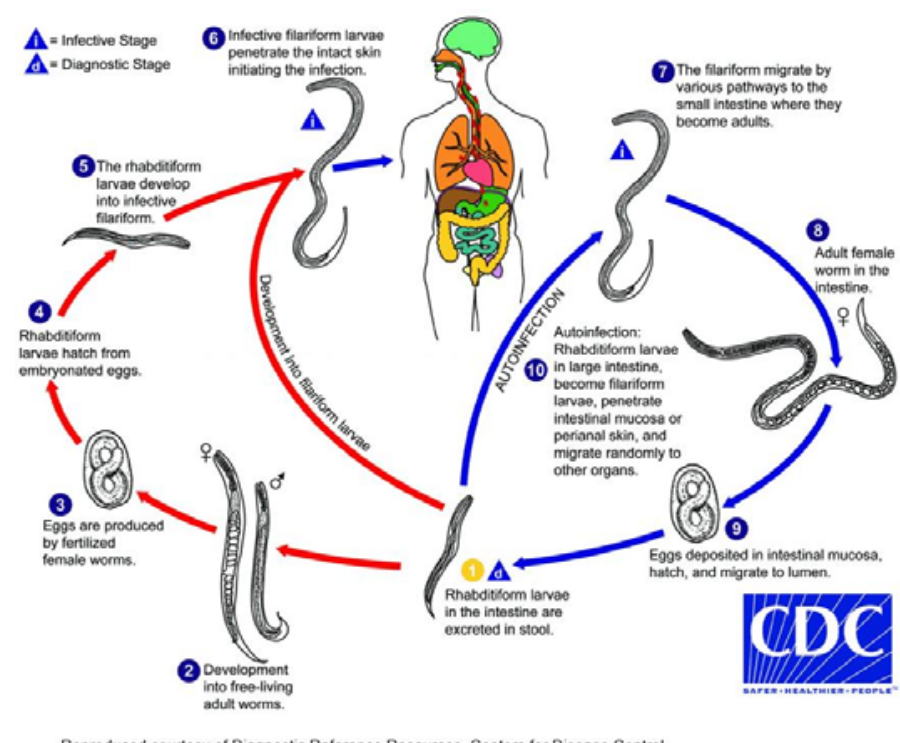

Figure 1: Life cycle of Strongyloides stercoralis ${ }^{6}$. 


\section{Case report}

An Aboriginal woman aged 71 years was diagnosed with stage IV ovarian carcinoma in 2012 in north-western New South Wales, Australia. Initial surgery and partial liver resection was followed with palliative chemotherapy with oral etoposide. She remained asymptomatic until August 2015 when she developed tiredness and lethargy. The patient reported no abdominal pain. Her past history was unremarkable. She had no previous testing for strongyloidiasis. She did not drink alcohol and was a non-smoker.

Examination was normal except for conjunctival pallor. Her abdominal examination revealed her previous surgical scars. There was no tenderness or abdominal masses palpable. Bloods showed mild anaemia with haemoglobin $11.0 \mathrm{~g} / \mathrm{L}$ (normal range 11.5-16.5 g/L), macrocytosis (mean corpuscular volume $112.7 \mathrm{fL}$; normal range $80-100 \mathrm{fL}$ ) and white cells borderline elevated $11.4 \times$ $10^{9} / \mathrm{L}$ (normal range $4.0-11.0 \times 10^{9} / \mathrm{L}$ ). The white cell differential comprised neutrophils $6.5 \times 10^{9} / \mathrm{L}$ (normal range $1.8-7.7 \times 10^{9} / \mathrm{L}$ ), lymphocytes $1.2 \times 10^{9} / \mathrm{L}$ (normal range $1.0-4.0 \times 10^{9} / \mathrm{L}$ ) and monocytes $0.7 \times 10^{9} / \mathrm{L}$ (normal range $\left(0.1-0.8 \times 10^{9} / \mathrm{L}\right.$ ). Eosinophilia was $3 \times 10^{9} / \mathrm{L}$ (normal $<0.6 \times 10^{9} / \mathrm{L}$ ). Electrolytes and creatinine were normal. Her gamma glutamyltransferase was elevated, at $185 \mathrm{U} / \mathrm{L}$ (normal range (9-36 U/L). Other liver functions, albumin and bilirubin, were within the normal range. Imaging by chest X-ray and abdominal ultrasound failed to show any new recurrence of her ovarian cancer.

In view of her macrocytosis, tests for thyroid function, B12 and folic acid were ordered, which were normal.

In view of her Aboriginal heritage and eosinophilia, a travel history was taken. The women had lived in an unsewered area of the mid north coast of New South Wales during her youth. She had resided between coastal New South Wales and north-western New South Wales. There was no travel overseas or to northern Australia.

Strongyloides IgG enzyme immunoassay was undertaken. This comprised use of the Strongyloides L3 antigen (produced by DRG Diagnostics and offered by Laverty Pathology via QML Pathology in Australia). Her enzyme immunoassay was strongly positive (signal-to-cutoff ratio 2.910; normal <0.2).

Stool microscopy showed no eggs or larvae; culture with Koga agar plate method $\mathbf{~}^{\mathbf{7}}$ failed to hatch larvae from a stool sample. The patient was treated with albendazole $400 \mathrm{mg} \times 3$ days with improvement in symptoms and resolution of her eosinophilia to 0.1 (normal <0.6) after 6 weeks. Chemotherapy with oral etoposide was resumed. Several weeks later she developed left basal pneumonia with a recurrence of her tiredness. She was treated with intravenous antibiotics, and investigations showed a recurrence of eosinophilia $\left(1.4 \times 10^{9} / \mathrm{L}\right.$; normal $\left.<0.6 \times 10^{9} / \mathrm{L}\right)$. Treatment failure to albendazole was considered and confirmed by persistently raised Strongyloides serology. She was treated with ivermectin at recommended dose for weight, with resolution of symptoms. Her Strongyloides serology remained negative after treatment in 2015. During the episode of pneumonia, she was unable to produce a sputum sample for microscopy to search for
Strongyloides larvae.

She remained asymptomatic for another 12 months. She elected to cease chemotherapy and there was a recurrence of her ovarian carcinoma, with metastasis to her bowel. This was inoperable and she died of bowel obstruction.

\section{Discussion}

This case report highlights that strongyloidiasis may be contracted in temperate areas of Australia and can remain asymptomatic until immunosuppression arises later in life.

Two relatively unique adaptations of $S$. stercoralis, namely autoinfection and parthogenesis, mean that chronic strongyloidiasis can persist for years in a human host (Fig1). Common immunosuppressive conditions that have been associated with strongyloidiasis include HIV infection, use of steroids and chemotherapy ${ }^{4}$. The patient in the present study received etoposide, which has been associated with fulminant strongyloidiasis ${ }^{4}$.

Traditionally, strongyloidiasis is seen as a tropical disease in Australia with a distribution of north of latitude $23.5^{\circ}$ south $\mathbf{1}$. Other temperate regions, particularly temperate Russia and the Appalachian region of North America, have areas where strongyloides is and or was endemic. These regions are or were linked to poor sanitation and lower socioeconomic status ${ }^{\mathbf{1}}$. The warm, moist environment of the human intestine allows $S$. stercoralis larvae to survive over cold periods. When faecal contamination of warm soil occurs, a new generation of worms and larvae can colonise the soil, with transmission to other humans.

The woman in the present study resided in the mid north coast of New South Wales during her youth, in a town $31.91^{\circ}$ south, which has a high rainfall. She lived her adult life in an inland community of north-western New South Wales with a latitude of $30.73^{\circ}$, which is dry with high summer temperatures and cold winters. Moist, warm soils are needed to host Strongyloides larvae and adult worms. In this patient, there is some evidence to support the assumption that endemic infection has arisen in mid north coastal New South Wales. Cooper ${ }^{\mathbf{8}}$, in a program to manage diabetes in the Aboriginal communities of northern coastal New South Wales, extended screening to Strongyloides. Between 2004 and 2006, 62 of 194 patients tested positive for Strongyloides, a prevalence of $32 \%$. It is notable in Cooper's study that not all patients with positive Strongyloides serology had eosinophilia.

Eosinophilia as an inconsistent finding of chronic strongyloidiasis has also been observed in the Northern Territory $\mathbf{5 , 9}$. The patient described in the present case demonstrated other common observations in treatment. Treatment with albendazole over 3 days can be associated with treatment failure ${ }^{9}$. Ivermectin is now the treatment of choice ${ }^{\mathbf{5}}$. Monitoring treatment success should be conducted using Strongyloides serology titres to observe a decrease in titre rather than relying on a reduction in eosinophils alone ${ }^{9}$. The eosinophilic response may vary with the 
immunological competence of a patient and is not necessarily a marker of treatment success ${ }^{4}$. Similarly, an intact immune system is needed to produce antibodies to Strongyloides ${ }^{\mathbf{5}}$. It is possible to have a seronegative infection with faecal or sputum positive microscopy, PCR protocol or culture ${ }^{\mathbf{5}}$. Negative serology alone should not completely exclude Strongyloides in a person with a very high suspicion of risk ${ }^{5}$.

It is interesting that this patient developed respiratory symptoms when her chemotherapy was recommenced, with incomplete eradication of her strongyloidiasis. Some of her pneumonia-like symptoms may be attributed to larval migration to the lungs from increasing Strongyloides infection in the bowel.

Within Australia, calls have been made to make strongyloidiasis a notifiable disease. By doing this it is hoped to highlight the prevalence of disease and improve control measures ${ }^{\mathbf{1 0}}$. Poor sanitation can still occur in remote and rural areas of Australia, with Strongyloides serology prevalence rates of greater than $25 \%$ recorded in surveys $\mathbf{~}^{\mathbf{1 1}}$. The present case adds further evidence to suggest transmission can extend to the temperate areas of
Australia; similar temperate transmission has been noted previously in Russia and the Appalachian areas of the USA ${ }^{\mathbf{1}}$.

\section{Conclusion}

A high index of suspicion, together with the need to take a detailed family and travel history, is needed when considering whether a patient's symptoms could be caused by strongyloidiasis $^{\mathbf{5}}$. This patient's Aboriginal heritage and eosinophilia raised clinical suspicions to investigate for this disease. Strongyloidiasis is not confined to the Aboriginal population alone. Returned service personnel and migrants from endemic areas are other groups at higher risk due to their travel overseas $^{\mathbf{1}}$. The present case adds evidence to suggest strongyloidiasis may have been contracted in temperate areas of Australia in the past when sanitation was poor.

\section{Acknowledgements}

I acknowledge the late Professor Richard Speare, who was an inspiration during my years of training and encouraged the publication of this case report.

\section{REFERENCES:}

1 Beknazarova M, Whiley H, Ross K. Strongyloidiasis: a disease of socioeconomic disadvantage. International Journal of Environmental Research and Public Health 2016; 13(517). https://doi.org/10.3390/ijerph13050517 PMid:27213420

2 Yamada M, Matsuda S, Nakazawa M, Arizono N. Series-specific differences in heterogonic development of serially transferred freeliving generations of Strongyloides planiceps and Strongyloides stercoralis. Journal of Parasitology 1991; 77(4): 592-594. https://doi.org/10.2307/3283165 PMid:1865267

3 Conway D, Lindo J. Robinson R, Bundy D. Towards effective control of Strongyloides stercoralis. Parasitology Today 1995; 11(11): 420-424. https://doi.org/10.1016/0169-4758(95)80023-9

4 Keiser P, Nutman T. Strongyloides stercoralis in the immunocompromised population. Clinical Microbiology Reviews 2004; 17(1): 208-217. https://doi.org/10.1128

/CMR.17.1.208-217.2004 PMid:14726461

5 Page W, Speare R. Chronic strongyloidiasis - don't look and you won't find. Australian Family Physician 2016; 45(1): 40-44.

6 Centre for Disease Control Diagnostic Reference Resources. Lifecycle of Strongyloides stercoralis. Available: https://www.cdc.gov
/dpdx/images/strongyloidiasis/Strongyloides_LifeCycle2015.gif (Accessed 31 March 2019).

7 Koga K, Kasuya S, Khamboonruang C, Sukhavat K, leda M, Takatsuka N, et al. A modified agar plate method for detection of Strongyloides stercoralis. American Journal of Tropical Medicine and Hygiene 1991; 45(4): 518-521. https://doi.org/10.4269 /ajtmh.1991.45.518 PMid:1951861

8 Cooper J. Strongyloidiasis in northern NSW. Proceedings of 4th National Strongyloidiasis Workshop. Adelaide, 12 July 2007.

9 Page W, Dempsey K, McCarthy J. Utility of serological follow-up of chronic strongyloidiasis after anthelminthic chemotherapy. Transactions of the Royal Society of Tropical Medicine and Hygiene 2006; 100: 1056-1062. https://doi.org/10.1016 /j.trstmh.2005.12.006 PMid:16551471

10 Speare R, Miller A, Page W. Strongyloidiasis: a case for notification in Australia? Medical Journal of Australia 2015; 202(10): ii. https://doi.org/10.5694/mja15.00112 PMid:26021359

11 Adams M, Page W, Speare R. Strongyloidiasis: an issue in Aboriginal communities. Rural and Remote Health 2003; 3: 152. Available: http://rrh.org.au/journal/article/152 (Accessed 26 May 2016).

This PDF has been produced for your convenience. Always refer to the live site https://www.rrh.org.au/journal/article/4787 for the Version of Record. 